\title{
Linear Sigma Model at Finite Baryonic Density and Symmetry Breakings
}

\author{
F. L. Braghin \\ Instituto de Física, Universidade de São Paulo; C.P. 66.318; CEP 05315-970; São Paulo, Brazil.
}

Received on 29 September, 2006

\begin{abstract}
The linear sigma model at finite baryonic density with a massive vector field is investigated considering that all the bosonic fields develop non zero expected classical values, eventually associated with condensates and corresponding to dynamical symmetry breakings which might occur in the QCD phase diagram. A modified equation for the classical vector field is proposed with its respective solution. Some in medium properties of the model (mainly masses) are investigated within reasonable prescriptions. In particular the behavior of the in medium pion and sigma masses and a particular way of calculating in medium coupling to baryons is investigated. A symmetry radius for finite baryonic densities is proposed and calculated in different ways in terms of the other variables of the model and these different ways of calculating it agree quite well. However, assuming that the pion and sigma masses go to zero close to the restoration of chiral symmetry a too high value for the critical density is obtained $\rho_{c} \simeq 4.3 \rho_{0}$.
\end{abstract}

Keywords: Linear sigma model; Finite baryonic density; QCD phase diagram

\section{INTRODUCTION}

Matter at high energy densities has been continuously investigated to provide a deepeer understanding of strong interactions. Experimental (relativistic and high energy) heavy ion collisions (r.h.i.c. and h.e.h.i.c.) provide very important collection of data to construct this knowledge. Asymptotic freedom is a key property in this program. With different approaches, hadronic models based on quantum chromodynamics (QCD) at finite energy density have been extensively investigated and different condensates are usually expected to appear at different energy densities. In the vacuum, the lightest strong interacting particles are known to respect, approximatedly at least, chiral symmetry $S U_{L}(2) \times S U_{R}(2)$ which is expected to be spontaneously broken down to $S U(2)$. This is expected to give rise to a scalar (quark-antiquark) condensate, which might have relevant effects in experimental conditions and in realistic calculations, it rearranges the theory [1-3]. As a consequence, the hadronic properties which depend on this condensate (which can be the order parameter or proportionally to it [4]) are expected to vary with energy density. In particular the behavior of the rho vector meson has been considered as a possible signature of the chiral symmetry restoration via dilepton emission [5] although recent experimental analysis of the rho vector meson spectral function shows to be incompatible with energy shift [7].

In this work the Linear Sigma Model (LSM) [8] at finite baryonic density, $\rho_{B}$, is investigated with a massive classical vector field. It is based in $[9,10]$. All the mesons in the model are considered to develop classical counterparts. There is a renewed interest in the pseudoscalar condensation indeed $[9,11,12]$. It provides a way of enhancing $\mathrm{CP}$ violation at finite density [13]. The exact field equations and the stability equation are truncated such as analytical solutions are obtained by considering particular prescriptions for the stability condition. The numerical solutions have a self consistency although the so-called "full self consistency" is only achieved in a level of approximation and for the interactions considered in a model, not in the complete self consistency of the exact re- alistic quantum theoretical many body problem which still is too difficult to obtain. The truncations in the effective action, done in the next section, are based in the following considerations: (1) the effective potential of spin zero bosons keeps the same form of that at the tree level calculation (i.e., quantum fluctuations basically rearrange the tree level model), (2) each component of the system, i.e. baryons/ spin zero bosons/ spin one fields, have nearly independent stability conditions. Hopefully this assumption might go along with the observation of different slope parameters and temperature freeze-out [14] for each of the hadrons emerging from relativistic heavy ion collisions - and eventually different contributions for the corresponding Hydrodynamics. The corresponding (dynamical) equation for each of the fields is satisfied. The complete numerical investigation of the results will be presented elsewhere $[9,15]$. Several properties of in medium hadrons are investigated, namely scalar and pseudoscalar meson masses and couplings, and their relation to the behavior of the (chiral) symmetry is worked out within a particular prescription which provides results in agreement with the usually expected behavior. A symmetry radius is defined for the investigation of the symmetry behavior and its estimation (and dependence with the baryonic density) is done in several ways which yield very close result. In spite of being a quite simple model, without several degrees of freedom which should be relevant at high energy densities, results show to be consistent. Assuming that pion and sigma masses go to zero close to the density in which chiral symmetry would be restored a too high critical density is obtained. This can either signal that the model is too simple for describing Physics at too high densities or that their masses should not be expected to be zero close to the phase transition.

\section{THE LINEAR SIGMA MODEL AT FINITE $\rho_{B}$}

The Lagrangian density of linear sigma sodel (LSM) with baryons, $N_{i}(\mathbf{x})$, sigma and pions, $(\sigma, \pi)$, covariantly coupled 
to a vector field, $V_{\mu}$, is given by [8]:

$$
\begin{aligned}
& \mathcal{L}=\bar{N}_{i}(\mathbf{x})\left(i \gamma_{\mu} \tilde{\mathcal{D}}^{\mu}-g_{S}\left(\sigma+i \gamma_{5} \tau . \pi\right)\right) N_{i}(\mathbf{x}) \\
& +\frac{1}{2}\left(\mathcal{D}_{\mu} \sigma . \mathcal{D}^{\mu} \sigma+\mathcal{D}_{\mu} \pi \cdot \mathcal{D}^{\mu} \pi\right)+c \sigma+\frac{1}{2} m_{V}^{2} V_{\mu} V^{\mu}+ \\
& -\frac{1}{4} F_{\mu v} F^{\mu v}-\frac{\lambda}{4}\left(\sigma^{2}+\pi^{2}-v^{2}\right)^{2},
\end{aligned}
$$

where the covariant derivatives are given in [8] and they will not be completely considered in this communication. The other terms and parameters are standard [8, 9]. The introduction of a chemical potential, with an extra term $\delta \mathcal{L}=$ $-\bar{N} \gamma_{0} \mu_{\text {chem }} N$, is nearly equivalent to a shift of the classical temporal component of the vector field $V_{0}$ coupled to the nucleons. This field however is a dynamical degree of freedom (d.o.f.) and will be treated as such. Since the condensates (such as $\bar{\sigma} \equiv\langle\sigma\rangle$ ) depend on the density, so do most of the hadronic masses. Part of the baryon masses are considered to come from the the coupling to the scalar mesonic field and part from an explicit mass term for the baryons in the Lagrangian: $M^{*}=M \pm g_{S} \bar{\sigma}$.

The spin zero fields will be treated in the framework of the variational Gaussian approach with a truncation [3,9]. With the truncation of the effective potential of sigma and pion it can be written keeping the same form of the tree level effective potential. The equations for expected values of the sigma and pion are found accordingly, and shifts in these classical parts are considered due to the rearrangement brought by quantum fluctuations, such as $\bar{\sigma} \rightarrow \tilde{\sigma}$.

The total energy density is written in terms of the four variational parameters for the fields $\sigma, \bar{\pi}$, plus baryonic densities and vector field variables [9]. To investigate the behavior the temporal component of (classical) vector field, the total energy density is varied with respect to $V_{0}$, which is not quantized, instead of using its Euler-Lagrange equation. In this approach, $V_{0}$ is found either by writing a reasonable (or exact) expression for $\rho_{B}$ as a function of $V_{0}$, as it is given below, or $V_{0}$ is treated like a variational parameter such that one can determine a parametric function $\rho_{B}=\rho_{B}\left[V_{0}\right]$ which satisfy the equations of mouvement and stability. For this derivation, $\tilde{m}_{V}$ was kept constant. This second procedure yields a sort of variational equation for the corresponding parametric dependence. The corresponding (variational) equation for a constant background field component $V_{0}$ can be given by:

$$
\frac{\partial \mathcal{H}}{\partial V_{0}}=0 \rightarrow g_{V}\left(\rho_{B}+V_{0} \frac{\partial \rho_{B}}{\partial V_{0}}\right)-\tilde{m}_{V}^{2} V_{0}=0
$$

where the Euler-Lagrange equation for $V_{0}$ can be recovered by neglecting the derivative term above.

The stability condition for the ground state, with binding energy $E_{0} / A=\mathcal{H} / \rho_{B}<0$, can be written as $\frac{\partial \mathcal{H}}{\partial \rho_{B}}=$ $\left.\frac{\mathcal{H}}{\rho_{B}}\right|_{\rho_{B}=\rho_{0}}<0$, and $\left.\frac{\partial^{2} \frac{\mathcal{H}}{\rho_{B}}}{\partial \rho^{2}}\right|_{\rho_{B}=\rho_{0}}>0$, where $\rho_{0}$ is the stability density. The expressions for the energy density and its derivative with respect to $\rho_{B}$ is separated into three parts such that each component of the hadronic matter satisfies the stability equation above separatedly. With this prescription the solutions for the variational equations of each of the components satisfy the respective stability equation. The reliability of this factorization is not mathematically proven although some arguments for being reasonable are given in the Introduction and in [9]. The resulting equations (prescriptions) are the following:

$$
\begin{aligned}
& \text { (i) } \quad \frac{\partial E_{f}}{\partial \rho_{B}}=\frac{E_{f}}{\rho_{B}} ; \quad \text { (ii) } \quad \frac{\partial \mathcal{H}_{V}}{\partial \rho_{B}}=\frac{\mathcal{H}_{V}}{\rho_{B}} ; \\
& \text { (iii) } \frac{\partial\left(\tilde{\sigma}^{2}+\tilde{\pi}^{2}-v^{2}\right)}{\partial \rho_{B}}=\frac{\left(\tilde{\sigma}^{2}+\tilde{\pi}^{2}-v^{2}\right)}{2 \rho_{B}},
\end{aligned}
$$

Where $\mathcal{H}_{V}=g_{V} V_{0} \rho_{B}-\frac{1}{2} \tilde{m}_{V}^{2} V_{0}^{2}$. The complete set of solutions for the equations will be investigated elsewhere and compared to the exact numerical solutions. The variation of the sigma and pion masses arise from the corresponding classical fields. In the vacuum $\mu_{\pi}^{2}$ can go to zero as long as $\tilde{\pi}^{2} \rightarrow 0$ satisfying the Goldstone theorem when $c \rightarrow 0$ in the Lagrangian.

\section{A. Densities, coupling constants and masses}

The baryon fields, which depend on the bosonic fields through the Dirac equation coupled to the mesons, are quantized in terms of creation and annihilation operators. The baryonic degrees of freedom sum up into the densities: baryonic $\left(\rho_{B}\right)$, scalar $\left(\rho_{S}\right)$ and pseudo-scalar $\left(\rho_{p s}\right)$ densities. These quantitites will not be explicitely evaluated here although they are partially used below $[9,10]$. The energy density due to the fermions (antifermions) $\left(E_{f, \bar{f}}\right)$ and the density of baryons (antibaryons) $\left(\rho_{B, \bar{B}}\right)$ can be written, in the leading order, in terms of (1) their momenta for each kind of baryons $(i)$, up to the last occupied level with momentum $k_{F}$, and of (2) the classical vector field as $[10,16]$ :

$$
\begin{aligned}
& E_{(f, \bar{f})}^{i} \simeq \frac{\gamma}{(2 \pi)^{3}} \int^{k_{F}^{i}} d^{3} k\left(\frac{2 E_{(+,-)}^{i}\left(M_{i}^{*}+E_{(+,-)}^{i}\right)+V_{0}\left(V_{0}-2 E_{(+,-)}^{i}\right)}{2\left(M_{i}^{*}+E_{(+,-)}^{i}\right)}\right) \\
& \rho_{B, \bar{B}}^{i} \simeq \frac{\gamma}{(2 \pi)^{3}} \int^{k_{F}^{i}} d^{3} k\left(\frac{\left(M_{i}^{*}+E_{(+,-)}^{i}\right)^{2}-\mathbf{k}^{2}}{2 M_{i}^{*}\left(M_{i}^{*}+E_{(+,-)}^{i}\right)}\right)
\end{aligned}
$$

In these expressions $E_{ \pm}=g V_{0} \pm \sqrt{k^{2}+\left(M^{*}\right)^{2}}$ are the eigenvalues of the corresponding Dirac equation $[10,16]$. These expressions still correspond to an approximation and show deviations from the Fermi liquid picture.

The scalar and pseudoscalar densities, which appear in the equations of $\sigma, \bar{\pi}$, can be expanded in terms of the scalar and pseudoscalar condensates, for example, as:

$$
\begin{aligned}
& \rho_{S}=\rho_{S}^{(0)}+\frac{\tilde{\sigma}}{\tilde{\sigma}_{v a c}} \rho_{S}^{(1)}+\frac{\tilde{\sigma}^{2}}{\tilde{\sigma}_{v a c}^{2}} \rho_{S}^{(2)}+\frac{\tilde{\sigma}^{3}}{\tilde{\sigma}_{v a c}^{3}} \rho_{S}^{(3)}+o(|\tilde{\pi}|), \\
& \rho_{P S}=|\tilde{\pi}| \rho_{P S}^{(1)}+\left|\tilde{\pi}^{2}\right| \rho_{P S}^{(2)}+\left|\tilde{\pi}^{3}\right| \rho_{P S}^{(3)}+o(\tilde{\sigma}),
\end{aligned}
$$

where the coefficients $\rho_{S, P S}^{(j)}$ are obtained from the expressions calculated with the solution of the corresponding Dirac equation. They are such that $\rho_{S}=\rho_{p s}=0$ when $\tilde{\sigma}=\tilde{\sigma}_{v a c}$ and $\tilde{\pi}=0$, with higher order terms are indicated by $o(|\tilde{\pi}|)$ and $o(\tilde{\sigma})$. By substituting these expressions into the variational equations for the respective condensates [9] it is found that the terms proportional to $\tilde{\sigma}$ and $\tilde{\pi}$ can yield contributions to the in 
medium masses of sigma and pions. The terms proportional to $\tilde{\sigma}^{3}$ and $\tilde{\pi}^{3}$ can produce (effective) contributions for the coupling constant, i.e., $\lambda \rightarrow \lambda^{*}=\lambda \pm \frac{\rho_{S}^{(3)}}{\tilde{\sigma}_{\text {yac }}^{3}} \simeq \lambda \pm \rho_{P S}^{(3)}$. These two corrections for the in medium effective coupling constant may also be different from each other, eventually leading to different interactions of the pion and sigma in the baryonic medium.

The second equation of prescriptions (3) can produce the same solution of the equation (2). The third of prescriptions (3) can define a symmetry radius in the medium:

$$
\left(\tilde{\sigma}^{2}+\tilde{\pi}^{2}-v^{2}\right)=\tilde{C} \sqrt{\rho_{B}} .
$$

In this expression $\tilde{C}$ is a constant to be determined from the parameters of the model. In the vacuum: $\tilde{\sigma}^{2}=v^{2}=f_{\pi}^{2}$ as discussed above. Modifications in the equation (3-(iii)) will produce different dependences on the baryonic density. A more general symmetry radius, corresponding to particular modifications of the corresponding differential equation due to diverse couplings for example, might be written as: $\left(\tilde{\sigma}^{2}+\tilde{\pi}^{2}-v^{2}\right)=\left(\tilde{D}+\tilde{C} \rho_{B}{ }^{c}\right)^{\gamma}$ where $\tilde{D}, \tilde{C}, c, \gamma$ are constants to be related to the parameters of the model.

One way of calculating $\tilde{C}$ is found by assuming that this symmetry radius is valid over a range of baryonic densities. This is a crude approximation because, heavier hadrons as well as quark and gluon d.o.f. are expected to be relevant for high energy densities. In the high density when chiral symmetry should be restored: $\tilde{\sigma}=\tilde{\pi} \rightarrow 0$. This critical density is written as $\rho_{c}=u \rho_{0}$. . Thus in this point: $\tilde{C}=\mp \tilde{v}^{2} \sqrt{\frac{1}{u \cdot \rho_{0}}}$. At the saturation density $\left(\rho_{B}\right)$ the expression for $\tilde{C}$ can be written as:

$$
\tilde{\sigma}^{2}+\tilde{\pi}^{2} \simeq \tilde{v}^{2}\left(1 \pm \sqrt{\frac{1}{u}}\right)=\left(f_{\pi}^{0}\right)^{2}\left(1 \pm \sqrt{\frac{\rho_{B}}{\rho_{c}}}\right) .
$$

Four values are considered: (i) $u=2$, (ii) $u=3$, (iii) $u=3.5$ and (iv) $u=4$. Considering the branch of solutions for which $\tilde{\sigma}^{2}+\tilde{\pi}^{2}<\tilde{v}^{2}$, at $\rho_{B}=\rho_{0}$, it follows respectively:

$$
\begin{aligned}
& \left.\sqrt{\tilde{\sigma}^{2}+\tilde{\pi}^{2}}\right|_{\rho_{0}} \simeq 0.54 \tilde{v}(i),\left.\quad \sqrt{\tilde{\sigma}^{2}+\tilde{\pi}^{2}}\right|_{\rho_{0}} \simeq 0.65 \tilde{v}(i i), \\
& \left.\sqrt{\tilde{\sigma}^{2}+\tilde{\pi}^{2}}\right|_{\rho_{0}} \simeq 0.68 \tilde{v}(i i i),\left.\quad \sqrt{\tilde{\sigma}^{2}+\tilde{\pi}^{2}}\right|_{\rho_{0}} \simeq 0.71 \tilde{v}(i v) .
\end{aligned}
$$

Other solutions are not presented. Since the squared value $\bar{\pi}^{2}$ is a scalar which appear very often in the expressions, it may be that $f_{\pi}^{*} \simeq \sqrt{\tilde{\sigma}^{2}+\tilde{\pi}^{2}}$, i.e., a pion classical field could be responsible for modifications in the pion decay constant and consequently measurable, even if competing with other effects. The topological Skyrme model can provide some argument in favor of such interpretation for a classical pion field inside hadrons [17]. These expressions may be therefore useful for relating descriptions of different ranges of the matter phase diagram.

The values obtained for $\tilde{C}$ from estimates (8) are respectively given by:

$$
\begin{aligned}
& \tilde{C} \simeq \pm 0.41 \mathrm{fm}^{-\frac{1}{2}}(i), \quad \tilde{C} \simeq \pm 0.33 \mathrm{fm}^{-\frac{1}{2}}(i i), \\
& \tilde{C} \simeq \pm 0.30 \mathrm{fm}^{-\frac{1}{2}}(i i i), \quad \tilde{C} \simeq \pm 0.28 \mathrm{fm}^{-\frac{1}{2}}(i v) .
\end{aligned}
$$

Another way of estimating $\tilde{C}$ is shown by considering the meson masses in the medium. With the expressions for meson masses in terms of the classical fields and $v[8,9]$, the symmetry radius can be written as: $\tilde{C} \sqrt{\rho_{B}}=\frac{1}{4 \lambda}\left(\left(\mu_{T}^{*}\right)^{2}-\left(\mu_{T}^{\text {vac }}\right)^{2}\right)$, where $\left(\mu_{T}^{(*)}\right)^{2}=\left(\mu_{S}^{(*)}\right)^{2}+\left(\mu_{P}^{(*)}\right)^{2}$ at a given density $\rho_{B}$. In these expressions the coupling $\lambda$ was also kept constant (and positive) and $c=0$, in the Lagrangian term. Two possible behaviors are obtained in this picture for the restoration of chiral symmetry: the sum of these masses may decrease or increase depending on the sign of $\tilde{C} \sqrt{\rho_{B}}$. For $\rho_{0}=0.15 \mathrm{fm}^{-3}$ and $\tilde{C} \simeq-0.15 \mathrm{fm}^{-\frac{1}{2}}$ the above expression yields approximated values $\left(\mu_{T}^{*}\right)^{2}\left(\rho_{0}\right) \simeq(1 \pm 0.53) \mu_{T}^{2}\left(\rho_{B}=0\right)$. If one considers that the pion and sigma masses disappear close to the chiral symmetry restoration point (i.e., if $\left(\mu^{*}\right)^{2} \rightarrow 0$ ), with the values above we obtain that $\rho_{c} \simeq 4.3 \rho_{0}$. Seemingly it is a too high baryonic density and the reasons are quite apparent. Firstly, as emphasized above, the present work only takes into account the light sector of hadrons and it does not consider quark and gluon degrees of freedom. Furthermore, it is a controversial subject whether pion and sigma masses (two point Green's functions) should be expected to be so close to zero (as it was assumed to obtain such high value for the critical density) close to (and at the) deconfinement critical point.

These ways of calculating $\tilde{C}$ provide crude (but interesting and curious) estimations. The corresponding in medium hadron properties are qualitatively in agreement with other estimations [5]. The inclusion of other relevant d.o.f. will be presented elsewhere as well as a corresponding calculation at finite temperature.

\section{B. Summary and Conclusions}

In this work some aspects of the Linear Sigma Model at finite baryonic density were investigated with a massive classical vector field, based in $[9,10]$. All the mesons in the model were considered to develop classical counterparts. In part this is due to independent new investigations on pseudoscalar condensates which have shown a renewed interest in the pseudoscalar condensation indeed [9, 11, 12]. The exact field equations and the stability equation were truncated for obtaining analytical solutions which capture the expected behavior of the system. These solutions have a self consistency although the so-called "full self consistency" is only achieved in a level of approximation and for the interactions considered in a model, not in the complete self consistency of the exact realistic quantum theoretical many body problem which still is too difficult to obtain. The truncations in the effective action, done in the next section, are based in the following considerations: (1) the effective potential of spin zero bosons keeps the same form of that at the tree level calculation (i.e., quantum fluctuations basically rearrange the tree level model), (2) each component of the system, i.e. baryons/ spin zero bosons/ spin one fields, have nearly independent stability conditions. Hopefully this assumption might go along with the observation of different slope parameters and temperature freeze-out for each of the hadrons emerging from relativistic heavy ion 
collisions - and eventually different contributions for the corresponding hydrodynamic. The corresponding (dynamical) equation for each of the fields are satisfied. The complete numerical investigation of the results will be presented elsewhere $[9,15]$. Several properties of in medium hadrons were investigated, namely scalar and pseudoscalar meson masses and couplings, and their relation to the behavior of the (chiral) symmetry is worked out within a particular prescription which provides results in agreement with the expected behavior. A symmetry radius was defined for the investigation of the symmetry properties and its estimation (and dependence with the baryonic density) is done in several ways with fair agree- ment, in spite of being a quite simple model, without several degrees of freedom which should be relevant at high energy densities. Related aspects to matter-antimatter asymmetry in relativistic heavy ion collisions and in the Early Universe will be discussed and investigated elsewhere [16].

\section{Acknowledgements}

This work was partially supported by FAPESP, Brazil. F.L.B. thanks brief discussions with L.McLerran, R. Rapp, D. Zschiesche, H. Stoecker, R. Pisarski, M. Munhoz and G. Krein.
[1] S. Weinberg, The Quantum Theory of Fields -II, Cambridge University Press, (1996).

[2] F.L. Braghin, Doctoral thesis, (1996) unpublished

[3] P.M. Stevenson, B. Alles, and R. Tarrach, Phys. Rev. D 352407 (1987). F.L. Braghin, Phys. Rev. D 57, 3548 (1998); Phys. Rev. D 64, 125001 (2001). F.L. Braghin and F.S. Navarra, Phys. Lett. B 508, 243 (2001).

[4] H. Umezawa, Advanced Field Theory, AIP Press, (1995). J. Stern, Nucl. Phys B-Proc. Suppl. 64, 232 (1998).

[5] T. Hatsuda and T. Kunihiro, nucl-th/0112027. G. Chanfray, M. Ericson, and P.A.M. Guichon, Phys. Rev. C 63, 055202 (2001). D. Roder, J. Ruppert, and D.H. Rischke, Phys. Rev. D 68016003 (2003). J. Wambach, Prog. in Part. and Nuc. Phys. 50615 (2003). R. Rapp and J. Wambach, Adv. Nucl. Phys. 25, 1 (2000). D. Jido, T. Hatsuda, and T. Kunihiro, Phys. Rev. D 63, 011091 (2001). J. Delorme, G. Chanfray, and M. Ericson, Nuc. Phys. A 603, 239 (1996). L.S. Celenza, H.S. Wang, and C.M. Shakin, Phys. Rev. C 63, 025209 (2001). G.E. Brown and M. Rho, Phys. Rep. 269, 333 (1996). N. Kaiser, S. Fritsch, and W. Weise, Nuc. Phys. A 697, 255 (2002). P.K. Panda and F.L. Braghin, Phys. Rev. C 66, 055207 (2002).

[6] K. Suzuki et al, Phys. Rev. Lett. 92, 072302 (2004); K. Yokokawa, T. Hatsuda, A. Hayashigaki, and T. Kunihiro, hep$\mathrm{ph} / 0204163$.

[7] Damjanovic for the NA60 Collaboration, arXiv: nuclex/0609026 (2006).

[8] S. Gasiorowicz and D.A. Geffen Rev. of Mod. Phys. 41, 531
(1969).

[9] F.L. Braghin, pré-print IF-1570/2001; hep-ph/0206107; IF2006. F.L. Braghin, in HADRON Physics 2002, ed. by C. Vasconcellos et al World Scientific (2002).

[10] F.L. Braghin, Int. Journ. of Mod. Phys. D 13, 1267 (2004). F.L. Braghin, accepted to be published in Int. Journ. of Mod. Phys. D.

[11] H. Shen, S. Tamenaga, H. Toki, nucl-th/0409057.

[12] A.B. Migdal et al, Phys. Rep. 192, 179 (1990).

[13] See, for example, Y. Mori, K. Saito Phys. Lett. B 552, 21 (2003).

[14] For example in: F. Grassi, Braz. J. Phys. 35, 52 (2005) and references therein. HADRON-RANP Joint Meeting, RJ, Brazil, 2004, ed. by M. Bracco et al, AIP Conference Proceedings 739, APS (2004).

[15] F.L. Braghin, pre-print IF-USP (2005).

[16] F.L. Braghin, works presented in: ISMD 2006, Paraty, RJ, Brazil, September 2006; Physics in Collisions, Buzios, RJ, Brazil, July 2006 -e-Proceedings: arXiv:hep-ph/0609242; 18 th International Conference on Few Body, Santos, SP Brazil, August 2006.

[17] For example: T.H.R. Skyrme, Proc. Roy. Soc. Ser. A 260, 127 (1961). G.S. Adkins, in Chiral Solitons ed. by G.S. Adkins, Add. Wesley, 1988. F.L. Braghin, I.P. Cavalcante, Phys. Rev. C 67065207 (2003) and references therein. 